\title{
A DIRECT EXPANSION PROOF OF SUFFICIENT CONDITIONS FOR THE NON-PARAMETRIC PROBLEM OF BOLZA*
}

\author{
BY
}

WILLIAM T. REID

1. Introduction. In a recent paper $†$ the author has proved by expansion methods a sufficiency theorem for the general problem of Bolza that had been established previously by Hestenes $\ddagger$ using the classical field method. This proof had in common with the previous proof of Hestenes the property that the theorem was not established directly for the general problem of Bolza with non-separated end conditions; instead, such a problem was first transformed into an equivalent one in a greater number of dependent variables and with separated end conditions. In a paper appearing in the present volume of these Transactions, Hestenes $\S$ has devised a direct proof by field methods for the general problem of Bolza. He has utilized the notion of families of fields of extremals, and the proof is a generalization of a method which he has previously used in treating the problem of Mayer.

It is the purpose of the present note to give a direct expansion proof of sufficient conditions for the general non-parametric problem of Bolza. In this direct expansion proof the auxiliary theorem of $\$ 3$ of $\mathbf{R}$ is replaced by Theorem A of $\$ 2$ of the present paper. This latter theorem is an extension of the results of $\$ 5$ of $\mathrm{H}$ for the special case of a problem having the form of the second variation, and thus may be considered as an extension of the transformation of Clebsch.

It is to be remarked that there already existed direct sufficiency proofs by field methods for the general problem of Bolza in case suitable normality conditions were satisfied.|| Hestenes obtains in $\mathrm{H}$ a direct proof not involving normality assumptions. On the other hand, the results of the author's paper $\mathbf{R}$ do not yield a direct expansion proof of sufficient conditions for a problem with non-separated end conditions that involves no differential side condi-

\footnotetext{
* Presented to the Society, April 10, 1937; received by the editors January 20, 1937.

$\dagger$ Sufficient conditions by expansion methods for the problem of Bolza in the calculus of variations, to appear in a forthcoming number of the Annals of Mathematics. This paper will be referred to as $\mathbf{R}$.

$\ddagger$ These Transactions, vol. 36 (1934), pp. 793-818.

$\S A$ direct sufficiency proof for the problem of Bolza in the calculus of variations, these Transactions, vol. 42 (1937), pp. 141-154. This paper will be referred to as $\mathbf{H}$.

|| See, in particular, Morse, American Journal of Mathematics, vol. 53 (1931), pp. 517-546, and Bliss, Annals of Mathematics, vol. 33 (1932), pp. 261-274.
} 
tions, and which is thus identically normal. This fact is illustrated by the example given at the end of $\$ 3$ of $\mathbf{R}$. In view of this difference between the the generalization of Hestenes' paper $\mathbf{H}$ and the extension of the present note over the previous paper $\mathbf{R}$, it is somewhat surprising that essentially the same auxiliary theorem is involved in these two direct proofs by quite different methods of the same sufficient conditions for the general problem of Bolza.

2. Auxiliary theorems. The form of the problem of Bolza here considered is that of finding in a class of arcs

$$
y_{i}(x) \quad\left(i=1, \cdots, n ; x_{1} \leqq x \leqq x_{2}\right)
$$

satisfying differential equations and end-conditions

$$
\begin{aligned}
\phi_{\beta}\left[x, y, y^{\prime}\right] & =0 & (\beta=1, \cdots, m<n), \\
\psi_{\mu}\left[x_{1}, y\left(x_{1}\right), x_{2}, y\left(x_{2}\right)\right] & =0 & (\mu=1, \cdots, p \leqq 2 n+2),
\end{aligned}
$$

one which minimizes a given functional

$$
J=g\left[x_{1}, y\left(x_{1}\right), x_{2}, y\left(x_{2}\right)\right]+\int_{x_{1}}^{x_{2}} f\left[x, y, y^{\prime}\right] d x .
$$

The notation and terminology of $\mathbf{R}$ will be used throughout.

Suppose that $E: y_{i}(x), \lambda_{0}=1, \lambda_{\beta}(x)\left(x_{1} \leqq x \leqq x_{2}\right)$ is a non-singular extremal which satisfies with constants $e_{\mu}$ conditions I and IV ${ }_{*}^{\prime}$. This latter condition is that along $E$ the second variation

$$
J_{2}[\xi, \eta] \equiv 2 \gamma\left[\xi_{1}, \eta\left(x_{1}\right), \xi_{2}, \eta\left(x_{2}\right)\right]+\int_{x_{1}}^{x_{2}} 2 \omega\left[x, \eta, \eta^{\prime}\right] d x
$$

be positive for all non-identically vanishing variations $\xi_{1}, \xi_{2}, \eta_{i}(x)$ which satisfy the linear differential equations and end-conditions

$$
\begin{array}{ll}
\Phi_{\beta}\left[x, \eta, \eta^{\prime}\right] \equiv \phi_{\beta y_{i}} \eta_{i}+\phi_{\beta r_{i}} \eta_{i}^{\prime}=0 & (\beta=1, \cdots, m), \\
\Psi_{\mu}\left[\xi_{1}, \eta\left(x_{1}\right), \xi_{2}, \eta\left(x_{2}\right)\right] & \\
\quad \equiv \Psi_{\mu \xi_{1}} \xi_{1}+\Psi_{\mu \eta_{i_{1}} \eta_{i}}\left(x_{1}\right)+\Psi_{\mu \xi_{2}} \xi_{2}+\Psi_{\mu \eta_{i 2}} \eta_{i}\left(x_{2}\right)=0 & (\mu=1, \cdots, p) .
\end{array}
$$

As usual, it is assumed that the matrix $\left(\Psi_{\mu \xi_{1}} ; \Psi_{\mu \eta_{i 1}} ; \Psi_{\mu \xi_{2}} ; \Psi_{\mu \eta_{i 2}}\right)$ is of rank $p$. As a consequence of $\mathrm{IV}_{*^{\prime}}$ there exists a family $\eta_{i}=u_{i k}(x), \zeta_{i}=v_{i k}(x)$ $(k=1, \cdots, n)$ of mutually conjugate solutions of the canonical accessory equations with $\left|u_{i k}(x)\right| \neq 0$ on $x_{1} x_{2} . \dagger$

† See Reid, American Journal of Mathematics, vol. 57 (1935), pp. 573-586; also Morse, these Transactions, vol. 37 (1935), pp. 147-160, and Hestenes, Annals of Mathematics, vol. 37 (1936), pp. 543-551. 
Now suppose that $b_{\theta}{ }^{1}, a_{i \theta}{ }^{1}, b_{\theta}{ }^{2}, a_{i \theta}{ }^{2}(\theta=1, \cdots, 2 n+2-p)$ are linearly independent solutions of the equations

$$
\Psi_{\mu \xi_{1}} b_{\theta}^{1}+\Psi_{\mu \eta_{i 1}} a_{i \theta}^{1}+\Psi_{\mu \xi_{2}} b_{\theta}^{2}+\Psi_{\mu \eta_{i 2}} a_{i \theta}^{2}=0 \quad(\mu=1, \cdots, p) .
$$

Let $q$ denote the maximum number of linearly independent accessory extremals of the form $Y_{i l}(x) \equiv 0, Z_{i l}(x)(l=1, \cdots, q)$ such that for every nonidentically vanishing linear combination

$$
\eta_{i}=Y_{i l}(x) c_{l} \equiv 0, \quad \zeta_{i}=Z_{i l}(x) c_{l}
$$

the equations

$$
\zeta_{i}\left(x_{1}\right) a_{i \theta}^{1}-\zeta_{i}\left(x_{2}\right) a_{i \theta}^{2}=0 \quad(\theta=1, \cdots, 2 n+2-p)
$$

are not all satisfied. Without loss of generality we may then suppose that the sets $b_{\theta}^{s}, a_{i \theta}^{s}(s=1,2 ; \theta=1, \cdots, 2 n+2-p)$ are so chosen that

$$
Z_{i l}\left(x_{1}\right) a_{i \theta}^{1}-Z_{i l}\left(x_{2}\right) a_{i \theta}^{2}=-\delta_{l \theta}(l=1, \cdots, q ; \theta=1, \cdots, 2 n+2-p) .
$$

Then for an arbitrary accessory extremal $\eta_{i}(x) \equiv 0, \zeta_{i}(x)$ we have

$$
\zeta_{i}\left(x_{1}\right) a_{i \nu}^{1}-\zeta_{i}\left(x_{2}\right) a_{i \nu}^{2}=0 \quad(\nu=q+1, \cdots, 2 n+2-p)
$$

and there exists a unique accessory extremal $\eta_{i}=U_{i \nu}(x), \zeta_{i}=V_{i \nu}(x)$ such that

$$
U_{i \nu}\left(x_{s}\right)=a_{i \nu}^{s} \quad(s=1,2 ; \nu=q+1, \cdots, 2 n+2-p) .
$$

Let

$$
U_{i l}(x)=\rho Y_{i l}(x) \equiv 0, \quad V_{i l}(x)=\rho Z_{i l}(x) \quad(l=1, \cdots, q),
$$

where $\rho$ is a real constant whose value will be determined shortly. The multipliers corresponding to $u_{i k}, v_{i k}$ and $U_{i \theta}, V_{i \theta}$ will be denoted by $l_{\beta k}(x)$ and $L_{\beta \theta}(x)(k=1, \cdots, n ; \theta=1, \cdots, 2 n+2-p)$, respectively.

Suppose $\xi_{1}, \xi_{2}$ are constants and $\eta_{i}(x)$ are arbitrary functions of class $D^{\prime}$ on $X_{1} X_{2}$. Then there exist unique constants $c_{\mu}{ }^{\prime}, c_{\theta}(\mu=1, \cdots, p ; \theta=1, \cdots$, $2 n+2-p)$ such that

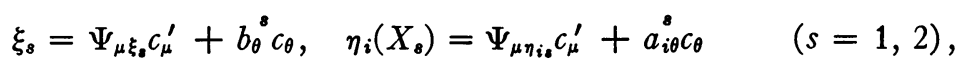

and corresponding to these constants $c_{\theta}$ there are determined for $X_{1}, X_{2}$ sufficiently near $x_{1}, x_{2}$ unique functions $h_{i}(x)$ of class $D^{\prime}$ and such that on $X_{1} X_{2}$

$$
\eta_{i}(x)=u_{i k}(x) h_{k}(x)+U_{i \theta}(x) c_{\theta} .
$$

For such a set $\xi_{1}, \xi_{2}, \eta_{i}(x)$ define

$$
\begin{aligned}
u_{i}^{\prime}(x) & =u_{i k}^{\prime}(x) h_{k}(x)+U_{i \theta}^{\prime}(x) c_{\theta}, \quad v_{i}(x)=u_{i k}(x) h_{k}^{\prime}(x), \\
l_{\beta}(x) & =l_{\beta k}(x) h_{k}(x), \quad L_{\beta}(x)=L_{\beta \theta}(x) c_{\theta}, \quad \mu_{\beta}=l_{\beta}+L_{\beta},
\end{aligned}
$$


and consider the expression

$$
2 \gamma\left[\xi_{1}, \eta\left(X_{1}\right), \xi_{2}, \eta\left(X_{2}\right)\right]+\int_{X_{1}}^{X_{2}}\left(2 \Omega\left[x, \eta, \eta^{\prime} ; \mu\right]-F_{r_{i} r_{k} v_{i} v_{k}}\right) d x .
$$

In this expression it is to be understood that the coefficients of $\gamma$ are the values determined by the end-values of $E$, and do not depend upon $X_{1}$ and $X_{2}$. For $X_{1}, X_{2}$ near $x_{1}, x_{2}$ the expression (2.7) is well-defined. The integral in (2.7) is readily seen to be the Hilbert integral for the second variation involving slope functions and multipliers determined by the $3 n-p$ parameter family of accessory extremals given above, and evaluated along the curve $\eta=\left[\eta_{i}(x)\right]\left(X_{1} \leqq x \leqq X_{2}\right)$. One may also verify directly by the use of Taylor's formula and the transformation of Clebsch that for $X_{1}, X_{2}$ near $x_{1}, x_{2}$ the expression (2.7) is a quadratic form in $\left[\xi_{1}, \eta_{i}\left(X_{1}\right), \xi_{2}, \eta_{i}\left(X_{2}\right)\right]$ whose coefficients depend upon the end-values of $u_{i k}, U_{i \theta}, v_{i k}, V_{i \theta}$ at $X_{1}$ and $X_{2}$. For brevity this form will be denoted by $2 Q\left[\xi_{1}, \eta_{i}\left(X_{1}\right), \xi_{2}, \eta_{i}\left(X_{2}\right) \mid X_{1}, X_{2}\right]$. Now it may be proved that the parameter $\rho$ involved in the definition of $U_{i l}, V_{i l}(l=1, \cdots, q)$ can be chosen so large that $Q\left[\xi_{1}, \eta_{i}\left(x_{1}\right), \xi_{2}, \eta_{i}\left(x_{2}\right) \mid x_{1}, x_{2}\right]>0$ for every nonzero set $\left[\xi_{1}, \eta_{i}\left(x_{1}\right), \xi_{2}, \eta_{i}\left(x_{2}\right)\right]$ satisfying the equations $\Psi_{\mu}\left[\xi_{1}, \eta\left(x_{1}\right), \xi_{2}, \eta\left(x_{2}\right)\right]=0$ $(\mu=1, \cdots, p)$ [see $\mathbf{H}, \S 5$ ]. In the following discussion we shall suppose that $\rho$ has been so chosen. It is to be emphasized that the arc $\eta=\left[\eta_{i}(x)\right]$ has not been assumed to be differentially admissible, and hence $\xi_{1}, \xi_{2}, \eta_{i}\left(X_{1}\right), \eta_{i}\left(X_{2}\right)$ are independent variables. By continuity it then follows that there exists a positive $\delta$ such that for $\left|X_{s}-x_{s}\right|<\delta(s=1,2)$ and an arbitrary non-zero set $\left[\xi_{1}, \eta_{i 1}, \xi_{2}, \eta_{i 2}\right]$ satisfying

$$
\left|\Psi_{\mu}\left[\xi_{1}, \eta_{i 1}, \xi_{2}, \eta_{i 2}\right]\right|<\delta\left[\xi_{1}^{2}+\xi_{2}^{2}+\eta_{i 1} \eta_{i 1}+\eta_{i 2} \eta_{i 2}\right]^{1 / 2}
$$

we have

$$
Q\left[\xi_{1}, \eta_{i 1}, \xi_{2}, \eta_{i 2} \mid X_{1}, X_{2}\right]>0 .
$$

Consequently, by the use of the integral form of the remainder in the expansions of the functions $\psi_{\mu}$ about the values $\left[x_{1}, y_{i}\left(x_{1}\right), x_{2}, y_{i}\left(x_{2}\right)\right]$ (see $\mathbf{R}, \S 3$ ) we obtain the following theorem:

Theorem A. Suppose that for a problem of Bolza $E: y_{i}(x), \lambda_{0}=1, \lambda_{\beta}(x)$ $\left(x_{1} \leqq x \leqq x_{2}\right)$ is a non-singular extremal which satisfies with constants $e_{\mu}$ conditions I and IV *'. Using the accessory extremals $u_{i k}, v_{i k}, U_{i \theta}, V_{i \theta}$ and terminology introduced above, there exists a neighborhood $\mathfrak{N}_{0}$ of the end-values $\left[x_{1}, y_{i}\left(x_{1}\right), x_{2}, y_{i}\left(x_{2}\right)\right]$ such that if $\left[X_{1}, Y_{i 1}, X_{2}, Y_{i 2}\right]$ is in $\mathfrak{N}_{0}$ the extremal $y_{i}(x), \lambda_{0}=1, \lambda_{\beta}(x)$ is defined and non-singular on $X_{1} X_{2}$ while $\left|u_{i k}\right| \neq 0$ on this interval; moreover, there exists a $\kappa>0$ such that if $\left[X_{1}, Y_{i 1}, X_{2}, Y_{i 2}\right]$ is in $\mathfrak{N}_{0}$ and terminally admissible we have 


$$
\begin{aligned}
& Q\left[X_{1}-x_{1}, \eta_{i 1}, X_{2}-x_{2}, \eta_{i 2} \mid X_{1}, X_{2}\right] \\
& \geqq \kappa\left[\left(X_{1}-x_{1}\right)^{2}+\left(X_{2}-x_{2}\right)^{2}+\eta_{i 1} \eta_{i 1}+\eta_{i 2} \eta_{i 2}\right],
\end{aligned}
$$

where $\eta_{i s}=Y_{i s}-Y_{i}\left(X_{s}\right)(s=1,2)$.

By relatively slight modifications of the proof of Theorem 4.3 of $\mathbf{R}$ one may establish the following corresponding result.

Theorem B. Suppose that $E: y_{i}(x), \lambda_{0}=1, \lambda_{\beta}(x)\left(x_{1} \leqq x \leqq x_{2}\right)$ is an extremal satisfying conditions $\mathrm{II}_{N}^{\prime}$, $\mathrm{III}^{\prime}$, and that $u_{i k}(x), v_{i k}(x), U_{i \theta}(x), V_{i \theta}(x)$ $(k=1, \cdots, n ; \theta=1, \cdots, 2 n+2-p)$ are associated accessory extremals with $\left|u_{i k}(x)\right| \neq 0$ on $x_{1} x_{2}$. For arbitrary sets $\left(h_{i}, c_{\theta}\right)$ let

$$
\begin{aligned}
y_{i}(x, h, c) & =y_{i}(x)+u_{i k}(x) h_{k}+U_{i \theta}(x) c_{\theta}, \\
r_{i}(x, h, c) & =y_{i}^{\prime}(x)+u_{i k}^{\prime}(x) h_{k}+U_{i \theta}^{\prime}(x) c_{\theta}, \\
\lambda_{\beta}(x, h, c) & =\lambda_{\beta}(x)+l_{\beta k}(x) h_{k}+L_{\beta \theta}(x) c_{\theta},
\end{aligned}
$$

where $l_{\beta k}$ and $L_{\beta \theta}$ are the multipliers for the accessory extremals $u_{i k}, v_{i k}$ and $U_{i \theta}, V_{i \theta}$, respectively. Then for every $\epsilon>0$ there exists $a d_{1 \epsilon}>0$ and a neighborhood $\mathfrak{F}_{1 \epsilon}$ in $x y$-space of the $[x, y(x)]$ on $E$ such that if $0 \leqq\left\|\left(c_{\theta}\right)\right\| \leqq d_{1 \epsilon}$, while $[x, y(x, h, c)]$ is in $\mathfrak{F}_{1 \mathrm{\epsilon}}$ and $[x, y(x, h, c), \tilde{r}]$ is differentially admissible, we have

$$
\begin{aligned}
\varepsilon[x, y(x, h, c), r(x, h, c), \lambda(x, h, c) ; \tilde{r}] & \\
& \geqq \tau R[\|\tilde{r}-r(x, h, c)\|]-\epsilon\left\|\left(h_{i}, c_{\theta}\right)\right\|^{2},
\end{aligned}
$$

where $\tau$ is a positive constant independent of $\epsilon . \dagger$

As in Theorem 4.3 of $R$ we may choose $\tau=\tau_{3} / 4$, where $\tau_{3}$ is the constant appearing in Lemma 4.3. In the above expression (2.8) $R[t]$ is the convex function $\left(1+t^{2}\right)^{1 / 2}-1$ for $t \geqq 0$.

Finally, we shall use Theorem 5.1 of $\mathbf{R}$, which for completeness we shall state.

THEOREM C. If $z_{t}(x)(t=1, \cdots, N)$ are absolutely continuous functions on $X_{1} \leqq x \leqq X_{2}$, and $\|z\| \leqq \delta$ on this interval, then

$$
\begin{array}{r}
\int_{X_{1}}^{X_{2}}\|z\| \cdot\left\|z^{\prime}\right\| d x \leqq d_{1}\left[\int_{X_{1}}^{X_{2}} R\left[\left\|z^{\prime}\right\|\right] d x+\left\|z\left(X_{1}\right)\right\|^{2}\right], \\
\int_{X_{1}}^{X_{2}}\|z\|^{2} d x \leqq d_{2}\left[\int_{X_{1}}^{X_{2}} R\left[\left\|z^{\prime}\right\|\right] d x+\left\|z\left(X_{1}\right)\right\|^{2}\right],
\end{array}
$$

where

$$
d_{1}=4\left(\left(1+\delta^{2}\right)^{1 / 2}+1\right) \max \left\{1, X_{2}-X_{1}\right\}, \quad d_{2}=3\left(X_{2}-X_{1}\right) d_{1} .
$$

$\dagger$ If $z \equiv\left(z_{t}\right)(t=1, \cdots, N),\|z\|=\left\|\left(z_{t}\right)\right\|$ is used to denote the positive square root of $z_{1}^{2}+\cdots+z_{N}$. 
3. The sufficiency theorem. We shall now indicate briefly how the direct proof of the following sufficiency theorem differs from the proof previously given in $\mathbf{R}$.

Theorem 3.1. Suppose that $E: y_{i}(x), \lambda_{0}=1, \lambda_{\beta}(x)\left(x_{1} \leqq x \leqq x_{2}\right)$ is an extremal arc satisfying with constants $e_{\mu}$ conditions $\mathrm{I}, \mathrm{II}_{N}^{\prime}, \mathrm{III}^{\prime}$ and $\mathrm{IV}_{*}{ }^{\prime}$. Then there exists a neighborhood $\mathfrak{F}$ of $E$ in $x y$-space and a neighborhood $\mathfrak{N}$ of the ends of $E$ in $\left[x_{1}, y_{i 1}, x_{2}, y_{i 2}\right]$-space such that $J[C]>J[E]$ for every admissible arc $C$ in $\mathfrak{F}$ with ends in $\mathfrak{R}$ and not identical with $E$.

Suppose that $C: Y_{i}(x)\left(X_{1} \leqq x \leqq X_{2}\right)$ is an admissible arc with end-points $\left[X_{1}, Y_{i}\left(X_{1}\right), X_{2}, Y_{i}\left(X_{2}\right)\right]$ in the $\mathfrak{R}_{0}$ neighborhood of $\left[x_{1}, y_{i}\left(x_{1}\right), x_{2}, y_{i}\left(x_{2}\right)\right]$ defined by Theorem A. Let $\eta_{i}(x)=Y_{i}(x)-y_{i}(x)$ on $X_{1} X_{2}$, and determine constants $c_{\mu}{ }^{\prime}, c_{\theta}(\mu=1, \cdots, p ; \theta=1, \cdots, 2 n+2-p)$ by equations (2.5) for $\eta_{i}\left(X_{s}\right)$ thus defined and $\xi_{s}=X_{s}-x_{s}(s=1,2)$. The corresponding functions $h_{i}(x)$ determined by (2.6) are of class $D^{\prime}$ on $X_{1} X_{2}$. If for a given $\operatorname{arc} C$ of this type we set

$$
u_{i}^{\prime}(x)=u_{i k}^{\prime}(x) h_{k}(x)+U_{i \theta}^{\prime}(x) c_{\theta}, \quad v_{i}(x)=u_{i k}(x) h_{k}^{\prime}(x)
$$

we have, in the notation of Theorem B,

$$
\begin{gathered}
Y_{i}(x)=y_{i}(x, h[x], c), \quad y_{i}^{\prime}(x)+u_{i}^{\prime}(x)=r_{i}(x, h[x], c), \\
\lambda_{\beta}(x)+\mu_{\beta}(x)=\lambda_{\beta}(x, h[x], c) .
\end{gathered}
$$

Finally, let $m_{0}, M_{0}$ be positive constants such that for every element $\left[X_{1}, Y_{i 1}, X_{2}, Y_{i 2}\right]$ in $\mathfrak{N}_{0}$ we have $m_{0}\|h\| \leqq\left\|\left(u_{i k}[x] h_{k}\right)\right\| \leqq M_{0}\|h\|$ for arbitrary sets $h=\left(h_{i}\right)$.

We write for brevity

$$
\Delta g=g\left[X_{1}, Y\left(X_{1}\right), X_{2}, Y\left(X_{2}\right)\right]-g\left[x_{1}, y\left(x_{1}\right), x_{2}, y\left(x_{2}\right)\right] .
$$

Then

$$
\begin{aligned}
J[C]-J[E] & =\Delta g+\int_{X_{1}}^{X_{2}} F\left[x, Y, Y^{\prime}, \lambda+\mu\right] d x-\int_{x_{1}}^{x_{2}} F\left[x, y, y^{\prime}, \lambda\right] d x \\
& =\Delta g+J^{0}-J^{1}-J^{2}
\end{aligned}
$$

where

$$
\begin{array}{cc}
J^{s}=(-1)^{s} \int_{X_{*}}^{x_{s}} F\left[x, y, y^{\prime}, \lambda\right] d x & (s=1,2), \\
J^{0}=\int_{X_{1}}^{X_{2}} \varepsilon\left[x, Y, y^{\prime}+u^{\prime}, \lambda+\mu ; Y^{\prime}\right] d x+\left.F_{r_{i}}\left(X_{s}\right) \eta_{i}\left(X_{s}\right)\right|_{s=1} ^{s=2}
\end{array}
$$




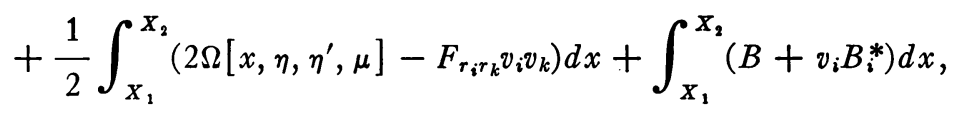

and for every $\epsilon>0$ there exists a corresponding $d_{\epsilon}>0$ such that if $\|\eta\| \leqq d_{\epsilon}$

$$
|B(x)| \leqq \epsilon\left\|\left\{h_{i}(x), c_{\theta}\right\}\right\|^{2}, \quad\left\|B^{*}(x)\right\| \leqq\left(\epsilon / M_{0}\right)\left\|\left\{h_{i}(x), c_{\theta}\right\}\right\| .
$$

The proofs of these relations are similar to those of (6.1), (6.2), (6.3) of $\mathbf{R}$. As in $R$, one may likewise show that for a given $\epsilon>0$ there is a bounded neighborhood $\mathfrak{R}_{2 \epsilon}$ of the end-points of $E$ which is interior to the $\mathfrak{N}_{0}$ neighborhood of Theorem A, and such that if $C$ is admissible with end-points in $\mathfrak{R}_{2 \epsilon}$, then

$$
\begin{aligned}
\Delta g & -J^{1}-J^{2}+\left.F_{r_{i}}\left(X_{s}\right) \eta_{i}\left(X_{8}\right)\right|_{s=1} ^{s=2} \\
& \geqq \gamma\left[X_{1}-x_{1}, \eta_{i}\left(X_{1}\right), X_{2}-x_{2}, \eta_{i}\left(X_{2}\right)\right] \\
& -\epsilon\left[\left(X_{1}-x_{1}\right)^{2}+\left(X_{2}-x_{2}\right)^{2}+\left\|\eta\left(X_{1}\right)\right\|^{2}+\left\|\eta\left(X_{2}\right)\right\|^{2}\right] .
\end{aligned}
$$

Let $\mathfrak{F}_{2 \epsilon}$ be a neighborhood of $E$ such that if $C$ is in $\mathfrak{F}_{2 \epsilon}$ then $\|\eta(x)\| \leqq d_{\epsilon}$ on $X_{1} X_{2}$. Consequently, if $C$ is an admissible arc in $\mathfrak{F}_{2 \epsilon}$ with end-points in $\mathfrak{R}_{2 \epsilon}$ we have $\dagger$

$$
\begin{aligned}
J[C]-J[E] \geqq & Q\left[X_{1}-x_{1}, \eta_{i}\left(X_{1}\right), X_{2}-x_{2}, \eta_{i}\left(X_{2}\right) \mid X_{1}, X_{2}\right] \\
& +\int_{X_{1}}^{x_{2}} \varepsilon\left[x, Y, y^{\prime}+u^{\prime}, \lambda+\mu ; Y^{\prime}\right] d x \\
& -\epsilon\left[\left(X_{1}-x_{1}\right)^{2}+\left(X_{2}-x_{2}\right)^{2}+\left\|\eta\left(X_{1}\right)\right\|^{2}+\left\|\eta\left(X_{2}\right)\right\|^{2}\right. \\
& +\int_{X_{1}}^{X_{2}}\left(\left\|\left\{h_{i}(x), c_{\theta}\right\}\right\|^{2}+\left\|\left\{h_{i}(x), c_{\theta}\right\}\right\| \cdot\left\|\left\{h_{i}^{\prime}(x), c_{\theta}^{\prime}\right\}\right\| d x\right] .
\end{aligned}
$$

For a given $\epsilon>0$ let $\mathfrak{F}_{\epsilon}$ denote a bounded neighborhood of $E$ in $x y$-space interior to both the neighborhood $\mathfrak{F}_{1 \epsilon}$ of Theorem $\mathrm{B}$ and the neighborhood $\mathfrak{F}_{2 \epsilon}$ defined above; moreover, suppose that $\mathfrak{N}_{\epsilon}$ is a neighborhood of the ends of $E$ interior to both $\mathfrak{N}_{0}$ and $\mathfrak{N}_{2 \epsilon}$, and such that if the end-values of $C$ are in $\mathfrak{N}_{\epsilon}$ the corresponding constants $c_{\theta}$ satisfy $\left\|\left(c_{\theta}\right)\right\| \leqq d_{1 \epsilon}$, where $d_{1 \epsilon}$ is as in Theorem B. Suppose $C: Y_{i}(x)\left(X_{1} \leqq x \leqq X_{2}\right)$ is an admissible arc in $\mathfrak{F}_{\epsilon}$ with endpoints in $\mathfrak{N}_{\epsilon}$. Since $\mathfrak{N}_{\epsilon}$ is interior to $\mathfrak{N}_{0}$ inequality (2.8) is applicable to the quadratic form $Q$. We also see that $\mathcal{E}\left(x, Y, y^{\prime}+u^{\prime}, \lambda+\mu ; Y^{\prime}\right]$ satisfies

† Since $c_{\theta}(\theta=1, \cdots, 2 n-p)$ are constants, and by Cauchy's inequality

$$
\|v\| \leqq M_{0}\left\|h^{\prime}(x)\right\|=M_{0}\left\|\left\{h_{i}^{\prime}(x), c_{\theta}{ }^{\prime}\right\}\right\|
$$

$$
\left|v_{i} B_{i}^{*}\right| \leqq \epsilon\left\|\left\{h_{i}^{\prime}(x), c_{\theta}^{\prime}\right\}\right\| \cdot\left\|\left\{h_{i}(x), c_{\theta}\right\}\right\| .
$$


(2.9). The functions $h_{i}(x)$ determined by $C$ are absolutely continuous, and $\left\|h^{\prime}(x)\right\|=\left\|\left\{h_{i}^{\prime}(x), c_{\theta}^{\prime}\right\}\right\|, R\left[\left\|h^{\prime}(x)\right\|\right], R[\|v(x)\|]$ are Riemann integrable on $X_{1} X_{2}$. Since $m_{0}\left\|h^{\prime}\right\| \leqq\|v\|$, we have $R\left[\left\|h^{\prime}\right\|\right] \leqq d_{3} R[\|v\|]$, where $d_{3}=\left(1 / m_{0}\right) \max$ $\left\{1,1 / m_{0}\right\}$ (see $\mathbf{R}$, Lemma A of $\$ 4$ ). Moreover, since $c_{\theta}(\theta=1, \cdots, 2 n+2-p$ ) are linear in $X_{s}-x_{s}, \eta_{i}\left(X_{s}\right)(s=1,2)$, there is a constant $m^{\prime}$ such that

$$
\left\|\left\{h_{i}\left(X_{1}\right), c_{\theta}\right\}\right\|^{2} \leqq m^{\prime}\left[\left(X_{1}-x_{1}\right)^{2}+\left(X_{2}-x_{2}\right)^{2}+\left\|\eta\left(X_{1}\right)\right\|^{2}+\left\|\eta\left(X_{2}\right)\right\|^{2}\right] .
$$

Applying (2.10) and (2.11) to the functions $\left(z_{t}\right)=\left(h_{i}, c_{\theta}\right)$ and combining the relations indicated above, it follows that for an admissible arc $C$ in $\mathfrak{F}_{\epsilon}$ with end-points in $\mathfrak{N}_{\epsilon}$, we have

$$
\begin{aligned}
J[C]-J[E] \geqq & \left(\kappa-\epsilon\left\{1+\left[2 d_{2}+d_{1}\right] m^{\prime}\right\}\right) \sum_{s=1}^{2}\left[\left(X_{s}-x_{s}\right)^{2}+\left\|\eta\left(X_{s}\right)\right\|^{2}\right] \\
& +\left(\tau-\epsilon\left[2 d_{2}+d_{1}\right] d_{3}\right) \int_{X_{1}}^{X_{2}} R[\|v(x)\|] d x .
\end{aligned}
$$

Now $d_{1}$ and $d_{2}$ depend upon the particular arc $C$ in that they involve $\left(X_{2}-X_{1}\right)$ and a quantity $\delta$ such that $\left\|\left\{h_{i}(x), c_{\theta}\right\}\right\|<\delta$ on $X_{1} X_{2}$. These quantities, however, are uniformly bounded when $\epsilon$ is restricted to a bounded set of values, and $C$ is in the $\mathfrak{F}_{\epsilon}$ neighborhood of $E$ with end-points in the $\mathfrak{N}_{\epsilon}$ neighborhood of the end-points of $E$. Hence the quantities $\left(\kappa-\epsilon\left\{1+\left[2 d_{2}+d_{1}\right] m^{\prime}\right\}\right)$, $\left(\tau-\epsilon\left[2 d_{2}+d_{1}\right] d_{3}\right)$ are positive for $\epsilon$ sufficiently small. Let $\mathfrak{F}=\mathfrak{F}_{\epsilon}, \mathfrak{N}=\mathfrak{N}_{\epsilon}$ for such a value of $\epsilon$. Then for an arbitrary admissible arc $C$ in $\mathfrak{F}$ with endpoints in $\mathfrak{N}$ we have $J[C] \geqq J[E]$, and the equality sign holds if and only if

$$
0=\int_{X_{1}}^{X_{2}} R[\|v(x)\|] d x=X_{s}-x_{s}=\left\|\eta\left(X_{s}\right)\right\| \quad(s=1,2),
$$

that is, if and only if $C$ is identical with $E$. We have established, therefore, the conclusion of Theorem 3.1.

As indicated in $\mathbf{R}$, the class of comparison arcs $C$ may be enlarged by interpreting the integrals involved as Lebesgue integrals.

The Institute for Advanced Study,

Princeton, N. J., AND

The University of Chicago, Chicago, Ill. 